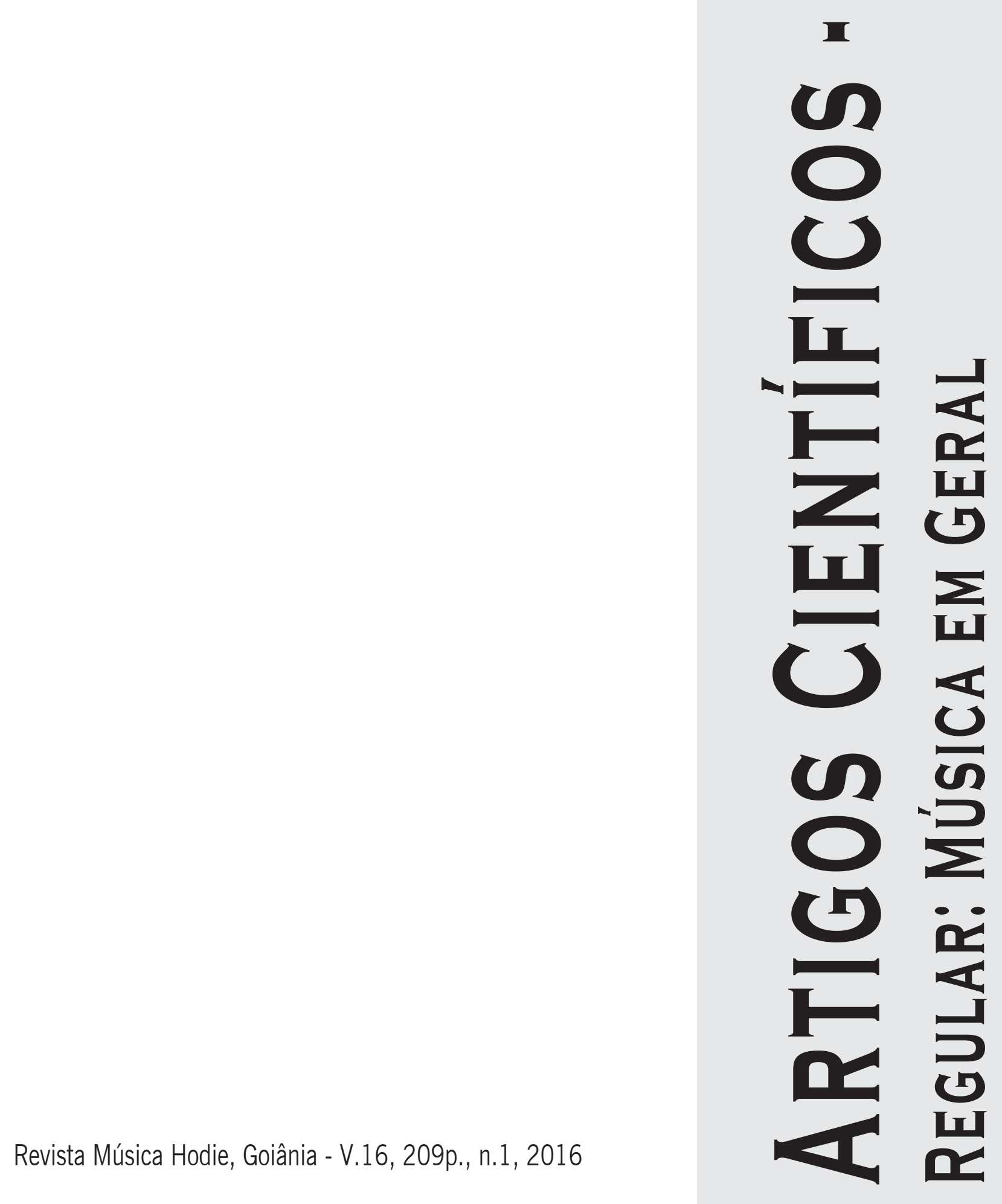




\title{
La Educación Musical en la escolaridad obligatoria durante el Franquismo. Un estudio a través de la legislación (1936-1982)
}

\author{
Consuelo Pérez-Colodrero (Universidad de Granada, Granada, Espanha) \\ consuelopc@ugr.es \\ Desirée García-Gil (Universidad Complutense de Madrid, Madrid, Espanha) \\ desiree.garcia@edu.ucm.es
}

\begin{abstract}
Resumen: Este trabajo indaga en la función que ejerció la enseñanza musical en la educación obligatoria española durante la época franquista según se desprende de la legislación orgánica (1936-1982). También se ocupa de la consideración que ha recibido tanto en el momento de ponerse en práctica como a partir de recientes investigaciones en historia de la educación. Al efecto, se vaciado el corpus legal sobre educación presente en el Boletín Oficial del Estado, realizándose un análisis cruzado entre dicha normativa y prensa periódica y literatura científica pertinente sobre el particular. Los resultados obtenidos sugieren que la enseñanza de la música se convirtió en uno de los medios óptimos para difundir la doctrina totalitaria entre la sociedad civil española, entendiéndose casi exclusivamente como canto colectivo y en el ámbito de la formación de la mujer.
\end{abstract}

Palabras clave: Educación musical española, Enseñanza obligatoria, legislación educativa, España (Periodo Franquista), Historia de la educación musical.

Music education in compulsory schooling during Francoism. A study through legislation (1936-1982)

Abstract: This paper enquires about the function of music education in Spanish compulsory schooling according to legislation promulgated between 1936 and 1982. It also argues the consideration these laws received at the time they were implemented and in recent researches on history of education. For this purpose, a comparative analysis amongst legal regulations, contemporary periodical press and relevant scientific literature has been carried out. The results obtained suggest that music education became one of the most relevant means in order to establish totalitarian doctrines amongst Spanish civil population, as it was conceived almost exclusively as collective singing and strongly emphasized in women education.

Keywords: Spanish music education, Compulsory education, Educational legislation, Spain (Francoism), History of music education.

A Educação Musical no ensino obrigatório durante o regime de Franco. Um estudo realizado através da legislação (1936-1982)

Resumo: Neste trabalho indaga-se sobre a função da educação musical no ensino obrigatório espanhol de acordo com a legislação promulgada entre 1936 e 1982. Ele também argumenta a consideração que essas leis recebeu no momento em que foram implementadas e em recentes pesquisas sobre a história da educação. Para este efeito, procedeu-se a uma análise comparativa entre as normas legais, a imprensa periódica contemporânea e a literatura científica relevante. Os resultados obtidos sugerem que a educação musical tornou-se um dos meios mais relevantes a fim de estabelecer doutrinas totalitárias entre a população civil espanhola, visto que foi concebida quase exclusivamente como canto coletivo e fortemente enfatizada na educação das mulheres.

Palavras-chave: Educação musical espanhola, Educação obrigatória, Legislação educacional, Espanha (Período Franquista), História da educação musical.

\section{Introducción}

La Guerra Civil española (1936-1939) truncó, junto a la armonía social y política, cualquier tipo de convivencia intelectual en el país ya que, según Díaz (2001), "la ideología oficial" que se impuso desde el comienzo fue una "amalgama” entre el conservadurismo tradicional y el totalitarismo del "ideario fascista” (p. 509). El vehículo de dicha intelectualidad, la educación, sufrió, junto a todos los demás pilares del Estado, la arremetida del régimen dictatorial, provocando la sumisión del país en una especie de oscurantismo general.

En efecto, según Peralta Ortiz (2012), la Junta de Defensa Nacional, ya desde el inicio de la sublevación armada, estaba muy interesada en hacer ver la normalidad de la vida 
nacional en los territorios ocupados, tomando como ejemplo el buen funcionamiento de la escuela. Así, la nueva política educativa se caracterizó por su escisión respecto a las medidas adoptadas en la Segunda República (1931-1939), pues éstas eran la consecuencia de la implantación de la que, a juicio del nuevo gobierno autoritario, era su partidaria e izquierdista ideología (JEFATURA DEL ESTADO [JE], 1945). La escuela, entonces, debía seguir los preceptos ideológicos del nuevo régimen y desligarse de lo "que se consideraba pernicioso para España y la educación” (p. 16), es decir, cualquier sombra de iniciativa republicana. No por casualidad, durante los primeros años de la dictadura se suceden reales decretos que continuamente auspician este nuevo orden educativo, en cualquier ámbito y nivel, en tanto que fue entendido como medio transmisor de la nueva ideología del Estado, según habrá ocasión de comprobar.

Partiendo de lo expuesto, este trabajo tiene por objeto [1] indagar en la función que ejerció la enseñanza musical en la educación obligatoria española del periodo Franquista, según se desprende de la legislación emitida entre el alzamiento militar de julio de 1936 y 1982, fecha de vigencia de la última ley franquista para la Educación, la Ley del 70. Al mismo tiempo, se pretende [2] argumentar la consideración que la educación musical tuvo dentro de la formación de una sociedad plural y cambiante y [3] determinar las concordancias y discrepancias que han existido entre la consideración que merecía cada ley en el momento de ponerse en práctica y en recientes investigaciones en educación musical de corte histórico.

Para ello, este trabajo toma como punto de partida la revisión legislativa de Oriol de Alarcón (2005) y las aportaciones realizadas por Rusinek y Sarfson (2010) y, utilizando una metodología histórico-descriptiva, responde a los objetivos planteados realizando un exhaustivo vaciado de Reales Decretos, Leyes Orgánicas y Órdenes educativas publicados en el Boletín Oficial del Estado, bajo cualquiera de sus denominaciones históricas, durante el período antes señalado (1936-1982). Los datos obtenidos se han complementando y contrastando con los provenientes de otras fuentes, principalmente de dos tipos: de un lado, publicaciones periódicas de alcance educativo y de especial interés para la horquilla de tiempo señalada, como la Revista de Pedagogía, o bien anuarios y colecciones legislativas del antiguo Ministerio de Instrucción Pública; de otro lado, se han revisado las aportaciones más relevantes al particular de parte de especialistas como López Casanova (2002a, 2002b) o Molero Pintado (1991), entre otros.

Las conclusiones obtenidas arrojan luz sobre el papel que la educación musical ejerció en uno de los momentos más aciagos de la historia española reciente, determinando cómo su uso sirvió al régimen totalitario como un vehículo portador de ideología.

\section{El canto patriótico como medio de ideologización: la educación musical en la legislación de la Nueva España (1938-1939)}

Desatada la Guerra Civil en julio de 1936, el bando sublevado fue promulgando normativas cuyo objeto era reanudar y organizar la enseñanza en las regiones ocupadas por el Ejército Nacional. El primer decreto en este sentido se destinó a consignar el restablecimiento de la escuela primaria, considerada la "piedra fundamental del Estado", en un explícito intento de "demostrar al mundo la normalidad de las regiones ocupadas" (PRESIDENCIA DE LA JUNTA DE DEFENSA NACIONAL [PJDN], 1936a, p. 35). Inmediatamente, se consignaron órdenes similares para la Educación Secundaria, en las que se hicieron patentes dos cambios con respecto a la regulación dimanada del anterior gobierno democrático: 
una fue la radical prohibición de la coeducación, en tanto que no estaba permitida la convivencia del alumnado de sexo masculino y femenino ni en el mismo aula ni en el mismo centro (PJDN, 1936b, p. 71); otra consistió en el adoctrinamiento político a través de la formación del individuo, tal y como se desprende de las siguientes líneas:

La gestión del Ministerio de Instrucción pública, y especialmente de la Dirección general de Primera Enseñanza, en estos últimos años, no ha podido ser más perturbadora para la Infancia. Cubriéndola con un falso amor a la cultura, ha apoyado la publicación de obras de carácter marxista o comunista, con las que ha organizado bibliotecas ambulantes y de las que ha inundado las escuelas a costa del Tesoro Público [...]. Es un caso de salud pública hacer desaparecer todas esas publicaciones [...]. Los Rectorados autorizarán, bajo su responsabilidad, el uso de obras cuyo contenido responda a los sanos principios de la Religión y de la Moral cristiana y que exalten con sus ejemplos el patriotismo de la niñez. (PJDN, 1936c, p. 72)

Un poco más adelante, se aprobaron las primeras instrucciones para la educación obligatoria en el Franquismo, que llegaron en marzo de 1938 merced a un texto que enunciaba los cuatro pilares indiscutibles sobre los que se asientaba la Educación Primaria - la Educación Religiosa, la Educación Patriótica, la Educación Cívica y la Educación Física - y en el que la música y la educación musical no se mencionan explícitamente. A este respecto, Pérez Zalduondo (2002) incide en el hecho de que la música se concibió "como apoyo de la labor formativa, pero en ningún caso como materia de enseñanza” (s.p. [apartado 2.2.1.4.]). La revisión de los textos jurídicos ha constatado fielmente dicho particular, advirtiéndose por un lado, la pluralidad de acciones acompañadas con música y, por otro lado, el cariz ideológico que cada una de estas actividades conllevaba. En este sentido, debe mencionarse, por ejemplo, que los niños debían entonar "cantos populares e himnos patrióticos [...] en todas las sesiones de la Escuela” (MINISTERIO DE EDUCACIÓN NACIONAL [MEN], 1938a, p. 6155) y, semejantemente, que "el acto de izar y arriar los días lectivos la enseña de la Patria en todas las escuelas nacionales, municipales y privadas" debía hacerse "mientras se canta[ba] el himno nacional”, que había de ser "obligatorio, dándosele toda la emoción necesaria” (ibídem). Así pues, podría apuntarse que, en este primer periodo, la actividad musical no se entendió como un fin en sí misma, sino que fue considerada como una herramienta de primer orden para inculcar al alumnado más joven la devoción a la patria y la consideración que el nuevo régimen quiso implantar de ella.

Apenas un mes más adelante, en abril de 1938, durante el primer gobierno regular del futuro cabeza de gobierno, Francisco Franco (El Ferrol, Galicia 1892-Madrid, 1975), aparecerá la primera articulación completa de la organización y las funciones de la educación obligatoria, vigente hasta 1945 (MEN, 1938d) y en la que tampoco se encuentra atisbo de educación musical ${ }^{1}$. No obstante es posible encontrar su clara incorporación en la reorganización que se hizo del Patronato de Misiones Pedagógicas en junio de 1939 que, en lo sucesivo, se denominaría "Patronato de Cultura Popular" y tendría por misión "difundir la cultura general, la moderna orientación docente y la educación ciudadana, en aldeas, villas y lugares, con especial atención a los intereses espirituales de la población” (MEN, 1939c, p. 3641). Ortiz Muñoz (1943), recogiendo la crónica de los primeros años de andadura de las Misiones, se congratulaba en aquel momento de que, en el corto plazo de tres años, se habían creado más de mil quinientas bibliotecas populares dotándolas de la necesaria orientación hacia la "catolización y la españolización" (p. 76). Dentro de esta, la música estuvo presente "como elemento importante en la educación de la infancia", haciéndose presente "en la Biblioteca en forma de historia, de canciones y de biografías" (ORTIZ MUÑOZ, 1943, 
pp. 76-77). Esta misma idea haya continuación en Escolar (1987), quien corrobora que, para atender a la finalidad básica de las Misiones, se organizaban "sesiones musicales de coros y pequeñas orquestas cuando fuera posible y, en todo caso, de audiciones de radiofonía y discos" (p. 29).

Según puede comprobarse, tanto en los primeros meses de la Guerra Civil como en los primeros años del Franquismo, el nuevo régimen se preocupó por legislar acerca de educación, si bien la música no gozó de atención específica en estos planes iniciales, en los que aparece como un medio y recurso más para impartir ideología y moldear al ciudadano, ahora súbdito de la Nueva España.

\section{La música puesta al servicio del Movimiento: la Ley sobre Educación Primaria de 1945}

Una vez asentada la dictadura del General Franco y tras un largo proceso de preparación y sistematización, la Ley sobre Educación Primaria de 1945, que había comenzado a gestarse en 1938, es aprobada el 14 de julio "con el voto en contra de diecinueve Procuradores” (Peralta Ortiz, 2012, p. 200). Rechazando de nuevo las propuestas de la República anterior, que "llevó a la Escuela una radical subversión de valores", prohibió la "imagen de Cristo" en los centros y encaminó a "la adolescencia al torvo empeño de la revolución marxista" (JE, 1945, pp. 385-386), la principal característica de esta nueva normativa para la educación obligatoria fue su sólida concepción católica, "principio inspirador [...] fundamental" de la misma, que se verificaba ya desde el mismo preámbulo:

La nueva Ley invoca entre sus principios inspiradores, como el primero y el más fundamental, el religioso. La Escuela española, en armonía con la tradición de sus mejores tiempos, ha de ser ante todo católica. Por eso, la Ley no vacila en recoger, acaso como ninguna otra en el mundo, y en algunos momentos con literalidad manifiesta, los postulados que consignó Pío XI como normas del derecho educativo cristiano en su inmortal encíclica Divini illius Magistri. De conformidad con ellas y con los principios del Derecho Canónico vigente, le corresponde para la educación por títulos de orden sobrenatural, y la potestad que le compete, cumulativamente con el Estado, de fundar Escuelas de cualquier grado, y, por tanto, Primarias y del Magisterio, con carácter de públicas, en armonía con la naturaleza jurídica de la Iglesia como sociedad perfecta y soberana. (p. 386)

Así, partiendo de las premisas asentadas por la ley, el sistema eclesiástico gozó de total libertad de actuación sobre la formación del individuo, de manera que Iglesia y Estado se constituyeron, en lo referente al adoctrinamiento patriótico y religioso, como dos caras de una misma moneda. Esta idea se vio reforzada con el precepto que estipulaba que debía "supedita[rse] la función docente a los intereses supremos de la Patria” (JE, 1945, p. 386), incluyéndose por ende, una nueva asignatura con un título marcadamente explícito, la "Formación del Espíritu Nacional”, que, según Peralta Ortiz (2012), denotaba una fuerte "inspiración cristiana” (p. 202). Otra de las singularidades de sus disposiciones fue, a emulación de la Ley de 1939 y en relación con lo que viene señalándose, la expresa prohibición de la coeducación, hecho manifiesto a lo largo de todo el documento, señalándose los ámbitos concretos a los que se circunscribía la actuación de la mujer - "la vida del hogar, artesanía e industrias domésticas” (JE, 1945, p. 388) -, apostándose por una marcada distinción entre competencias femeninas y masculinas.

Centrando las características de la Ley de 1945 en el tema de este trabajo, conviene empezar señalando que se dividió la enseñanza primaria en cuatro períodos, siendo única- 
mente obligatorios el segundo y el tercero de ellos, desde los seis a los doce años (JE, 1945, p. 389). El nuevo plan de estudios seccionó las materias de enseñanza en tres grupos (Tabla 1), de los que la formación musical se incluía en el denominado "Instrumental" a través de las asignaturas de "Música” y "Canto”. Además, la música estaba presente en las "actividades complementarias de la escuela", que comprendían la organización y celebración de "festivales con recitados, escenificaciones, conciertos, programas de radio y emisiones infantiles", supervisados, en el caso de los hombres por el Frente de Juventudes y, en el caso de las mujeres, por la Sección Femenina (JE, 1945, p. 395). Precisamente, esta última entidad fue la encargada de la formación Primaria y Secundaria de las mujeres, que incluía no solo la "Educación física”, la "Formación del Espíritu Nacional” sino, lo que es más importante para este estudio, "la práctica de la música y el baile tradicional” (MARTÍNEZ DEL FRESNO, 2010, p. 360). En otras palabras, el grupo femenino de la Falange debía ocuparse de la instrucción "política y social” de las mujeres (JE, 1939, p. 7347), transmitiendo el dogma fascista mediante un llamamiento al "cumplimiento de los deberes patrióticos" (RICHMOND, 2004, p. 43) a través de varias materias artísticas, entre las que la música hallaba un lugar destacado.

Tabla 1: Organización de las materiais de la Educación Primaria según el plan de estudios de 1945.

\begin{tabular}{|l|l|l|}
\hline \multicolumn{1}{|c|}{ Materias instrumentales } & \multicolumn{1}{|c|}{ Materias formativas } & \multicolumn{1}{c|}{ Materias complementarias } \\
\hline Lectura interpletativa & Formación religiosa & Iniciacións em las Ciencias de la Natureza \\
\hline $\begin{array}{l}\text { Expresión gráfica (escritura, ortografia, } \\
\text { redaccións y dibujo) }\end{array}$ & Formación del espiritu nacional & Materias artisticas (Música, Canto y Dibujo) \\
\hline Cálculo & $\begin{array}{l}\text { Formación intelectual (lengua nacional y ma- } \\
\text { temáticas) }\end{array}$ & $\begin{array}{l}\text { Materias utilitarias (trabajos manuales, prácticas } \\
\text { de taller y baores femeninas) }\end{array}$ \\
\hline & $\begin{array}{l}\text { Edicación fisica (fimnasia, deportes y juegos } \\
\text { dirigidos) }\end{array}$ & \\
\hline
\end{tabular}

Fuente: Elaboración propia a partir de JE (1945).

Castañón Rodríguez (2009) plantea el hecho de que la actuación del Movimiento convirtió la disciplina musical en "una especie de actividad de ocio, no muy definida, [...] impartida en un ámbito extraescolar y no formal, una maría de carácter lúdico [...] teñida con contenidos ideológicos" (p. 99). A pesar de ello, esta especialista pone de manifiesto la importante labor que la Sección Femenina (en adelante, SF) desempeñó en lo referente a la recuperación del repertorio tradicional, llegando a crear incluso una Regiduría especializada y dedicada, entre otros objetivos, a la "recogida de canciones y danzas en el medio rural”, la "organización de cursos especializados para la formación de instructoras", la conformación de los conocidos "grupos de coros y danzas - que "representa[ron] la imagen cultural de España en el extranjero" -, "la creación de subvenciones y ayudas para la innovación educativa en el área de la pedagogía musical" y muy especialmente "la elaboración de repertorios de canciones [...] con presencia paritaria de todas las regiones" (CASTAÑ́n RODRÍGUEZ, 2009, p. 104).

Es decir, la música, en tanto que materia de enseñanza, tampoco presentó un currículo definido en la ley de 1945, aunque fue parte fundamental en el adoctrinamiento político del individuo y, más concretamente, de la mujer. Este último cometido permite rastrear las razones para la insigne labor de recuperación y sistematización de repertorio folclorista por parte de la SF, que se materializó tanto en la elaboración de varios cancioneros como en la difusión del mismo a través de varios medios de propaganda (PÉREZ COLODRERO Y GARCÍA GIL, 2016a, 2016b). Dado lo expuesto, puede sugerirse que verdaderamen- 
te cumplió una misión pedagógica, en sentido amplio: no sólo se encargó de la formación de la mujer en diferentes y restrictivos niveles, sino que también se encargó de enseñar y fomentar en esta la merecida atención que requería la música a nivel social y cultural, teniendo ambas iniciativas como sustrato común la idea de relegar a la mujer a ámbitos muy concretos dentro de la sociedad: las féminas podrían encargarse de la música y de transmitir una serie de valores a través de ésta, pero no asumir un cargo de responsabilidad a otros niveles.

\section{Música es cosa de mujer: la ley de Ordenación de la Enseñanza Media de 1953}

La década de los cincuenta se tradujo en una cierta apertura ideológica y política del Franquismo: de un lado, a partir de 1950 el país empezó a insertarse en organismos internacionales - como la FAO (1950), la UNESCO (1952) o la ONU (1955) -, e iniciaba una política de acercamiento a los Estados Unidos - que permitió que empezara a recibirse asistencia económica del país norteamericano y que éste estableciera una serie de bases militares estratégicas en territorio español (SABÍN RODRÍGUEZ, 1997) -; de otro lado, el modelo autárquico fracasó, lo que conllevó un giro en la política económica, finalizando el racionamiento de alimentos y liberalizándose parcialmente los precios, el comercio y el tránsito de bienes (BARCIELA, 2003).

Semejante situación tuvo su influjo, como era natural, en el ámbito educativo. Joaquín Ruiz-Giménez Cortés (Hoyo de Manzanares, Madrid, 1913 - Madrid, 2009), responsable del Ministerio de Educación (ME), suscribió acuerdos con el Vaticano y con los Estados Unidos, de manera que, aunque persistieron la confesionalidad y el predominio de la Iglesia, la Ley de 26 de Febrero de 1953, sobre Ordenación de la Enseñanza Media, vio suavizado el peso que el patriotismo y el adoctrinamiento político tenían sobre lo técnico-pedagógico o, dicho de otro modo, el nuevo reglamento fue "menos dogmático y más atento a la calidad intelectual de la enseñanza” (MUÑOZ REPISO et al., 2000, p. 43).

Además el plan de estudios de 1953 supuso un nuevo paso hacia la generalización de la enseñanza, en tanto que establecía que "al menos su grado elemental” debía llegar a "todos los españoles aptos" (JE, 1953, p. 1120). Dicho nivel quedó dividido en dos grados, Elemental y Superior, de los que el primero constaba de cuatro cursos y se comenzaba a los diez años (JE, 1953, p. 1121) y el segundo tenía una duración de dos cursos y se iniciaba a los catorce años (JE, 1953, p. 1127). No obstante, el reglamento seguía manteniendo "el principio de una educación separada para los alumnos de uno y otro sexo” (JE, 1953, p. 1121), tanto desde el punto de vista de la organización como de la instrucción, pues quedaba estipulado que los centros femeninos debían preparar a las mujeres para "la vida del hogar" y para "profesiones femeninas" (JE, 1953, p. 1127), obligando entonces a que éstos se rigieran por un plan propio.

Precisamente, en la promulgación de 1953, la educación musical se comprendía solo en la formación femenina y, más concretamente, dentro de las "Enseñanzas del hogar", que la ley situaba de forma obligatoria "en los planes de todos los cursos, en los horarios escolares, en los exámenes y en las pruebas de Grado" (JE, 1953, p. 1127). El origen de este grupo de materias hay que buscarlo en el curso académico 1941/1942, año en el que su enseñanza, confiada a profesionales nombradas por la SF, se fija "en todos los centros de primera y segunda enseñanza, oficial y privada” (MEN, 1941a, p. 8090) y queda conformada por las disciplinas Economía doméstica, Labores, Corte, Trabajos manuales, Zurcido y repaso, Cocina y, finalmente, Música (MEN, 1941b). A partir del curso escolar 1944/1945, dichas 
materias, englobadas bajo la denominación genérica de "Escuelas del hogar”, fueron obligatorias en "todos los centros oficiales y privados de Enseñanza Media", durante "dos horas semanales por curso en los cinco primeros y de una hora y media semanal por curso para el sexto y séptimo respectivamente”, siendo además imprescindibles para que las estudiantes obtuvieran el título de Bachillerato (MEN, 1944, p. 6451).

De esta suerte, la ordenación de las enseñanzas medias de 1953 incluía la educación musical para las mujeres en el contexto concreto de su preparación para las actividades confiadas a su género. Pese a lo que esta situación pudiera poseer de negativo en un primer momento, de nuevo es Castañón Rodríguez (2009) quien subraya que la "obligatori[edad]" de formación musical en los institutos de Enseñanzas Medias durante los años cincuenta fue el primer paso para la formación de un "personal especializado", que se planteaba, además, con una marcada intención de "culturizar" a la mujer (p. 105). Con todo, resulta asimismo evidente que dicha formación tenía todavía unos tintes ideológicos y discriminativos muy marcados y que el sistema, pese a su voluntad aperturista, en cualquier caso, no contemplaba la educación musical como una de las materias básicas para educar y formar a todo individuo.

\section{Un primer intento de alfabetización musical: la Ley General de Educación de 1970}

La primera ley del pasado siglo que reguló y estructuró la totalidad del sistema educativo español fue la Ley General de Educación y Financiamiento de la Reforma Educativa del 4 de agosto de 1970, firmada por el ministro de Educación y Ciencia José Luis Villar Palasí (Valencia, 1922 - Madrid, 2012). Como último producto educativo de la Dictadura Franquista, esta ley, que quiso "superar las contradicciones internas en las que había caído el sistema por sucesivas reformas sectoriales” (MUÑOZ-REPISO et al., 2000, p. 44), no podía alejarse demasiado de los preceptos de la ideología del Régimen, pese a lo cual intentó adaptarse al acelerado proceso de cambio social y económico de la España del momento. Así, su artículo primero recoge que los "fines de la educación en todos sus niveles y modalidades" deberán ser "la formación humana integral, el desarrollo armónico de la personalidad y la preparación para el ejercicio de la libertad", siempre en "conformidad con lo establecido en los Principios del Movimiento Nacional” y de la Iglesia Católica (JE, 1970, p. 12527).

Puelles Benítez (2010) es uno de los especialistas que hace notar que existe una manifiesta contradicción de estos principios con la libertad de enseñanza que, recogida en diversos preceptos de la ley, debía ser sometida a lo establecido en el artículo mencionado. Aún más, dicho historiador entiende los preceptos liberales presentes en la ley ${ }^{2}$ como "una declaración velada del fracaso de treinta años de educación autoritaria”, resultándole muy llamativo el hecho de que para su redacción, además de "la experiencia de otros países europeos", se tuviera en cuenta el real decreto por el que se creaba el Instituto-Escuela en $1918^{3}$ (p. 339). De este modo, las diferencias entre la Ley de 1970 y las enunciadas en las décadas anteriores resultan claras: mientras las primeras se conformaron a partir de la ruptura con los criterios estipulados por ideologías apuestas al fascismo, la de 1970 nacía a partir de ellas, aunque el español medio no tendría forma ni medios para relacionarlas. Abundando en esta misma idea, tampoco puede obviarse la referencia que Villar Palasí hace a la Ley Moyano de 1857, que al tiempo ratifica el afán pseudo-democrático reflejado en la Ley de 1970: según el ministro, la inscripción de "nuestro sistema educativo [...] al esquema ya centenario" reflejaba un "estilo clasista" que no podía "atender a las necesidades" del país (JE, 1970, p. 12525). 
La Ley de 1970 solo plantea como obligatoria la Educación General Básica (EGB), que discurría desde los seis a los trece años de edad, si bien aquellos que no realizaran estudios superiores debían cursar obligatoriamente "una formación profesional de primer grado” (JE, 1970, p. 12527). El texto no hace referencia explícita a la Educación Musical, pero sí que refleja que "la formación se orientará", entre otros fines, "a la iniciación en la apreciación y expresión estética y artística” del alumnado (JE, 1970, p. 12529) ${ }^{4}$. Pérez Prieto (2001) llama la atención en torno a las carencias que planteaban las "orientaciones pedagógicas" de dicha ley, puesto que, a su juicio, éstas fueron meramente indicativas, en tanto que "tenían un marcado carácter experimental”, "la formulación de objetivos y contenidos [era] muy general" y no "ofre[cía] a los docentes instrumentos de apoyo" (p. 193). Es decir, aún siendo un primer intento para una regulación más abierta y plural que la estipulada en décadas anteriores, se vertebró como un banco de pruebas que seguía manteniendo la marca del régimen, descuidando los aspectos básicos del proceso de enseñanza-aprendizaje. En esta misma línea, Lorenzo Quiles (2003) subraya que, a pesar de que esta disciplina no fuera integrada entre los contenidos obligatorios del currículo, el ideario de educación integral reflejado para la EGB, hacía que la música se incluyera como parte "de una educación que deseaba ser completa” (p. 20). No obstante, los reales decretos y órdenes publicados con posterioridad regulando las materias de forma pormenorizada, que si bien ya se realizaron bajo un gobierno democrático, incidieron de manera decisiva en esta disciplina, según se puede comprobar a continuación.

La primera norma que concretó los contenidos de la ley general fue el Real Decreto 69/1981, que fijaba las enseñanzas mínimas para el Ciclo Inicial (MEC, 1981b) pero no daba cabida a la educación musical que, en cambio, sí que quedó recogida en la Orden de 17 de enero de 1981, regulando las enseñanzas de dicho ciclo (MEC, 1981a). Aunque no se da ningún tipo de indicación a nivel de concepción o metodología, el programa docente que se expone resulta en sí lo suficientemente significativo para entender la relevancia que la formación musical poseía en los primeros años de la educación obligatoria, que resulta un perfeccionamiento de la recibida a lo largo de la etapa de Preescolar (desde los 3 a los 5 años). Con una carga docente de tres horas semanales (MEC, 1981a, 1389), la música se concebía desde una perspectiva práctica y creativa, como medio de comunicación y expresión en el que el niño debía iniciarse y controlar en sus elementos básicos (rítmico, vocal y auditivo) (Tabla 2).

Así, la Orden del 6 de mayo de 1982, por la que se complementaban y desarrollaban las enseñanzas mínimas del ciclo medio de la EGB, incorporaba "los programas de las áreas de Educación Artística y de Educación Física” (MEC, 1982a, p. 12575). La primera de estas áreas "no [aspiraba] a conseguir especialistas, sino personas equilibradas y sensibles, capaces de captar y gozar de la belleza y constituirse, a su vez, en creadores” a través de la música, la plástica y el juego dramático: la Educación Musical se concibe de una manera activa y sensorial, buscando el desarrollo tanto de la creatividad como de la coordinación y el equilibrio de la acción gestual a través de "la articulación vocal, la práctica instrumental y el movimiento corporal” (MEC, 1982a, p. 12584). Al mismo tiempo, la música se entiende no de forma aislada, sino en correspondencia con las demás áreas, acrecentando de este modo su valor formativo en el desarrollo del individuo. Especial atención requiere el hecho de que la normativa legal potenciaba la práctica antes que la teoría, no solo por cuanto que así lo explicita el texto, sino porque, además, queda igualmente de manifiesto a partir de los cuatro bloques temáticos en los que se organizan los contenidos de la educación musical: "Expresión y comunicación a través de la música", "Música tradicional y colectiva", "Fuentes del sonido" y "Comportamiento del sonido en el lenguaje musical" (MEC, 1982a, p. 12584) (Tabla 2). 
Tabla 2: Contenidos de la educación musical de la EGB (ciclos inicial, medio y suérior).

\begin{tabular}{|l|l|l|}
\hline \multicolumn{1}{|c|}{ Ciclo Inicial } & \multicolumn{1}{|c|}{ Ciclo Medio } & \multicolumn{1}{c|}{ Ciclo Superior } \\
\hline Formación ritmica & Expresión y comunicación a través de la música & Expresión y comunicación a través de la música \\
\hline Educación vocal & $\begin{array}{l}\text { Música tradicional y colectiva } \\
\text { Fuentes del sonido }\end{array}$ & $\begin{array}{l}\text { Música tradicional y colectiva } \\
\text { Fuentes de sonido, agrupación de voces e instrumentos } \\
\text { más generalizados }\end{array}$ \\
\hline Educación auditiva & Comportamento del sonido em el lenguaje musical & Percepción de los elementos constitututivos de la música \\
\hline & & Música em la sociedad actual \\
\hline
\end{tabular}

Fuente: Elaboración propia a partir de MEC (1981a, 1982a, 1982b).

En contraposición, en el Ciclo Superior de la EGB, la Música fue considerada, en un primer momento, de manera inversa, es decir, como un contenido no práctico sino teórico. Así se desprende del Real Decreto 3087/1982 del 12 de noviembre de 1982 por el que se fijan las enseñanzas mínimas para dicha etapa educativa, en la que la disciplina se incluye en el bloque temático de Historia, a su vez comprendida dentro del área de Ciencias Sociales. En concreto, se esperaba que el alumnado conociese y comprendiese las características fundamentales del arte no solo a partir de sus obras arquitectónicas, pictóricas, escultóricas, sino también musicales (MEC, 1982c, p. 32014), lo que para Pérez Prieto (2005) hacía que la música "deja[se] de ser una materia de expresión artística" y pasase a estar "más cerca de la historia del arte y la estética” (p. 87).

Apenas quince días más tarde, se da un paso más con la regulación de las enseñanzas del Ciclo Superior, (Orden del 25 de noviembre de 1982, Ministerio de Educación y Ciencia, MEC, 1982b), que definitivamente incorpora los programas de Educación Artística y Educación Física, con los que complementa y desarrolla lo expuesto en el Real Decreto 3087/1982 (MEC, 1982a), dotando al área de música de mayor plasticidad, profundidad y contenido práctico. En este sentido, la principal diferencia respecto al Ciclo Medio estriba en la pretensión de dotar al alumno de conocimientos técnicos y teóricos que lo facultaran para entender de manera compleja un producto artístico de índole plástico, dramático o musical. Con respecto a éste último, la atención se centró en relacionar al alumno con su entorno sonoro natural y en "cultivar la sensibilidad como vehículo para la expresión de sentimientos y vivencias personales” (MEC, 1982a, p. 33463). Al mismo tiempo, se subrayó el estudio tanto de las "composiciones populares o clásicas" como de los "componentes musicales y los recursos específicos más comunes” en éstas (MEC, 1982a, p. 33463). Dado este planteamiento, los bloques temáticos quedaron divididos en cinco (Tabla II): "Expresión y comunicación a través de la música”, "La música tradicional y colectiva", "Fuentes de sonido, agrupación de voces e instrumentos más generalizados", "Percepción de los elementos constitutivos de la música" y "La música en la sociedad actual" (MEC, 1982a, p. 33463).

Al igual que ocurría en el Ciclo Medio de la EGB, también en el Ciclo Superior se daba potestad al profesor para distribuir los contenidos del modo que considerara necesario y siempre "en función de los intereses, necesidades y madurez de los alumnos" (MEC, 1982a, p. 33463).

Dado lo expuesto, es posible afirmar que la ley de 1970 posibilitó el inició de un currículo específico y completo para la formación musical, puesto que no solo hacía referencia a los contenidos de la disciplina, sino también a la metodología a emplear por parte del profesor. Esta última variable sería el auténtico talón de Aquiles de esta regulación educativa, por encima de otras deficiencias que sí que lograron salvarse, según queda expuesto y descrito por Roche Márquez (1994): 


\begin{abstract}
A pesar de lo incomprensible que nos resulta el constatar que la Música, dentro del ámbito escolar, carece de peso específico, es de destacar en la justificación que se hace del área de Expresión Dinámica en el nuevo Plan, la defensa de métodos que relacionen al niño con la música de forma directa y activa, la preocupación por desarrollar su capacidad creativa y la inclusión de la educación del movimiento, si bien la falta de claridad y orden en el desarrollo de los contenidos y la imprecisión de una buena parte de los objetivos específicos restan al conjunto eficacia y orientación. No ocurre así con los programas renovados aparecidos en abril de 1981, en donde la división en bloques temáticos y el desarrollo de los mismos ofrecen un material de indudable calidad y utilidad para el trabajo escolar que, una vez más, no pudo ser experimentado por la misma carencia de profesores capacita- dos para llevarlos a las aulas. (p. 7)
\end{abstract}

En consecuencia, la Ley de 1970 debe recordarse porque contribuyó a que se diera una verdadera alfabetización musical al alumnado en edad de escolarización obligatoria, ya que, de un modo gradual y a través de los dos últimos ciclos de la EGB, se potenciaba la acción y el entendimiento de sus elementos constitutivos, tanto teóricos como prácticos, por considerarlos parte imprescindible de la enseñanza global del individuo.

\title{
Conclusiones y consideraciones finales
}

La revisión legislativa e histórica ha permitido dar respuesta a los objetivos planteados al inicio del presente estudio, articulándose por tanto las conclusiones a raíz de éstos.

La enseñanza musical en la educación obligatoria española estuvo fuertemente vinculada al adoctrinamiento ideológico y a la inculcación de los principios vertebradores del régimen. La educación musical se convierte, en los primeros años de vigencia del gobierno del general Franco, en mero canto patriótico y en acompañamiento de las actividades claramente vinculadas a la exaltación de los símbolos nacionales. Con todo, conviene señalar ciertos aspectos relacionados con la educación musical en diferentes contextos sociales. Para empezar, es preciso subrayar la clara vinculación de la música con el género femenino, tanto porque la presencia de la educación musical es manifiesta en las asignaturas dedicadas en exclusiva a dicho género (dentro de las llamadas 'Enseñanzas del hogar') como por su relación, fomento y difusión a través de la Sección Femenina de la Falange y de las J.O.N.S., responsables, en cualquier caso, de la educación de la mujer, casi en exclusiva, durante los primeros años del Régimen Franquista. Además, debe ponerse en relieve la evolución que la disciplina experimentó durante el periodo de la dictadura, que responde, sin duda, a la propia evolución de ésta. Si durante los primeros años, según podía ocurrir con el resto de las artes y coincidiendo con la Autarquía y el mayor hermetismo ideológico, la música se centraba, según se ha indicado, en ser transmisora y reforzadora de la ideología y en estar al servicio de la Propaganda del régimen, durante los años cincuenta y sesenta se experimenta un cambio, que se corresponde con la llegada del Plan Marshall y los primeros indicios de apertura y que conlleva una progresiva liberalización de los tintes ideológicos que llegará a su cénit con la Ley de 1970.

Precisamente, esta última normativa constituyó el primer caso de alfabetización general del alumnado español y, más concretamente desde el ámbito musical, en tanto que, a pesar de las contradicciones en las que incurría la ley (PUELLES BENÍTEZ, 2010), aspiraba a formar "personas equilibradas y sensibles, capaces de captar y gozar de la belleza y constituirse, a su vez, en creadores” (MEC, 1982a, anexo I, p. 12584) a través tanto de la música como de la plástica y el juego dramático. La educación integral promovida a partir de las artes fue, sin duda, uno de los primeros logros en materia de educación musical en 
España, que con la Ley de 1970 dio un primer paso hacia los presupuestos que se habían implantado, con bastantes años de ventaja, en el resto de países del entorno europeo.

Parece claro que la educación musical ha gozado siempre de una consideración positiva para la formación y que los especialistas en educación se han mostrado en todo momento aseverativos respecto a las bondades y perversiones de cada sistema educativo. Según se ha mostrado a lo largo de las anteriores páginas, cabe destacar las aportaciones de Pérez Zalduondo (2002) y Castañón Rodríguez (2009), especialistas en música y educación musical durante el franquismo; Richmond (2004), cuya obra sobre la Sección Femenina de la Falange resulta indispensable para entender la instrucción de la mujer durante tal período y, Pérez Prieto (2001, 2005), especialista en la legislación desde 1970. Estos coinciden en señalar que la de dictadura prohibió la coeducación, que la enseñanza se imbuyó de carácter religioso y que se suprimieron las asignaturas de índole libertaria. Además, según los especialistas estudiados, la formación musical no contó con una metodología específica, convirtiéndose principalmente en vehículo transmisor de ideología y herramienta de primer orden para comunicar el amor a la patria y la lealtad al sistema político: queda así enmarcada dentro de la educación no formal, difundiendo un curriculum oculto, centrado en la transmisión de valores subversivos en contra de cualquier propuesta liberal.

Finalmente, debe tenerse en cuenta que, aunque inicialmente el gobierno de Franco circunscribiera la enseñanza musical de forma reglada casi en exclusiva al sexo femenino, a través de las "Enseñanzas del hogar", y a pesar de que dicha instrucción sirviera como medio de adoctrinamiento dentro de la línea ideológica del sistema - esto es, la mujer entendida principalmente en el ámbito de lo privado -, los historiadores mencionados han llegado a un punto común argumentando que, sin embargo, estas directrices se deben entender en positivo, pues se transcribieron en el primer intento de culturización de la mujer y de formación de un profesorado especializado (CASTAÑÓN RODRÍGUEZ, 2009).

De esta suerte, podría señalarse que la evolución que la educación musical experimentó durante el periodo de la Dictadura Franquista, responde, sin duda, al propio progreso de esta.

\section{Notas}

Esta legislación pretende recuperar las ideas de unidad y disciplina presentes en la Ley Moyano de 1857 (Ministerio de Fomento [MF], 1857) - dispersas, a juicio del legislador, en la multiplicidad de disposiciones reglamentarias que le siguieron -, a las que pretende añadir "los designios del nuevo Estado, nuestras realidades escolares y los caracteres genuinos de nuestra cultura y los progresos realizados hasta el día en materia pedagógica” (MEN, 1938d, p. 6763). Al efecto, la nueva normativa dispone que se constituya una Comisión, que debe encargarse de formar los nuevos programas de Educación Primaria y cuyos frutos se patentizarían, en primera lugar, en mayo de 1938, con la organización de cursos con las primeras "Orientaciones Nacionales para la Educación Primaria”, en cuyo programa de materias se incluyen el Sentido religioso y militar de la vida, la Educación política y nacional, la Organización social, la Metodología de la enseñanza infantil y la Educación física, pero no aparece la música (MEN, 1938c). Más adelante, en diciembre de 1938, la Comisión fijaría los programas escolares de primera enseñanza en una suerte de "ensayo pedagógico" que, ante todo, pretendía mostrar la nueva impronta, metodológica e intelectual, de la educación obligatoria en el territorio ocupado por el bando sublevado (MEN, 1938b, p. 3028), fundamentada en los "métodos de la España tradicional” y en el sistema de la repetición "clásico en la tradición española [...] para obtener la mayor fijeza y solidez de los conocimientos [pues] no hay que perder de vista que es preferible que éstos sean firmes y permanentes, aunque parcos, a que sean muchos y deleznables" (MEN, 1938e, p. 3036). Esta línea continuaría en la relación de libros aprobados para uso en Escuelas Nacionales de Primera Enseñanza en marzo de 1939 (MEN, 1939a), entre los que no se incluye nada relativo a música ni cancioneros pero sí que abundan los textos sobre doctrina católica y las lecturas patrióticas. Tampoco aparece referencia alguna a materiales musicales en la normativa que regulaba la adquisición de material pedagógico para las Escuelas Nacionales, entre el que se incluían mesas, bancos, mapas, atlas, crucifijos y estampas murales. No obstante, en un epígrafe se indica que también debe anunciarse concurso para la compra de "todo el res- 
to del material que se considere indispensable para el buen funcionamiento de las clases”, apartado en el que sí que podría darse por incluido el material musical que pudiera necesitarse para el normal desarrollo de la clase de Educación Primaria (MEN, 1939b, p. 3784).

2 En el preámbulo de Villar Palasí (JE, 1970) pueden leerse ciertos principios auspiciadores de un sistema de educación democrático: la ley proponía "oportunidades educativas a la totalidad de la población para dar así plena efectividad al derecho de toda persona humana a la educación” y debía "atender a la preparación especializada del gran número y diversidad de profesionales que requiere la sociedad moderna” (p. 12525).

3 El Instituto Escuela supuso la configuración de un sistema, muy similar al que se elaboraría posteriormente en época democrática, que promovía la formación integral del alumnado tanto en letras como en ciencias con atención a los principales estados del saber: Lengua castellana (lectura, escritura, gramática, composición y narración literaria), geografía, narraciones históricas, cálculos y nociones de Aritmética y Geometría, elementos de Ciencias de la Naturaleza, Legua Francesa, Caligrafía, dibujo y trabajos manuales, juegos, además de Excursiones, visitas a museos y lugares de interés. La instrucción musical de la "Sección Preparatoria" estaba presente en cada uno de los tres grados durante dos horas semanales a través de la asignatura "Música y Canto", cuyo concepto, dada su atención a la práctica vocal en grupo, fue casi equivalente al establecido en la Ley de Romanones; por su parte, en la "Sección Secundaria" la disciplina comprendería los cuatro primeros grados, asimismo durante dos horas semanales (MINISTERIO DE INSTRUCCIÓN PÚBLICA Y BELLAS ARTES, 1918, 164).

4 Fuera del ámbito obligatorio, la música corre igual suerte entre los contenidos de la Expresión Dinámica de la etapa de preescolar (LORENZO QUILES, 2003), aunque sí que estaba felizmente integrada en las materias comunes de Bachillerato (JE, 1970, p. 12529).

\section{Referencias}

BARCIELA, Carlos. Autarquía y mercado negro: el fracaso económico del primer franquismo 1939-1959. Barcelona: Crítica, 2003. 325 p.

CASTAÑÓN RODRÍGUEZ, Ma Rosario. El profesorado de educación musical durante el franquismo. Revista electrónica interuniversitaria de formación del profesorado, Madrid, v. 12, n. 4, 2009. Disponible en: <http://goo.gl/p5Fa8I>. Acceso el: 1 de octubre de 2015.

DÍAZ, Elías. Pensamiento político español del siglo XX. Pensamiento político bajo el régimen franquista (1939-1975). En: VALLESPÍN OÑA, Fernando (Ed.). Historia de la teoría política. Madrid: Alianza, 2001. p. 509-548.

ESCOLAR, Hipólito. La cultura durante la Guerra Civil. Madrid: Alhambra, 1987. 407p.

JEFATURA DEL ESTADO. Decreto sobre funciones de la Sección Femenina de Falange Española Tradicionalista y de las J. O. N. S. Boletín Oficial del Estado, Madrid, n. 363, de 29 de diciembre 1939, p. 7347-7348.

JEFATURA DEL ESTADO. Ley de 17 de julio de 1945 sobre Educación Primaria. Boletín Oficial del Estado, Madrid, n. 199, p. 385-416, 18 de julio de 1945.

JEFATURA DEL ESTADO. Ley de 26 de febrero de 1953 sobre Ordenación de la Enseñanza Media. Boletín Oficial del Estado, Madrid, n. 58, p. 1119-1130, 27 de febrero 1953.

JEFATURA DEL ESTADO. Ley 14/1970, de 4 de agosto, General de Educación y Financiamiento de la Reforma Educativa. Boletín Oficial del Estado, Madrid, n. 187, p. 12525-12546, 6 de agosto de 1970.

LÓPEZ CASANOVA, María Belén. La música en el Magisterio de las Escuelas Normales y su proyección a la primera enseñanza desde 1837 a 1930. Música y educación: Revista trimestral de pedagogía musical, Madrid, v. 15, n. 49, p. 29-44, 2002a.

LÓPEZ CASANOVA, María Belén. La política educativo-musical en España durante la Segunda República. Música y educación: Revista trimestral de pedagogía musical, Madrid, v. 15, n. 50, p. 15-26, 2002b. 
LORENZO QUILES, Oswaldo. Educación musical reglada en la enseñanza general española (1939-2002). Tavira. Revista de ciencias de la educación, Cádiz, n. 19, p. 13-34, 2003.

MARTÍNEZ DEL FRESNO, Beatriz. La sección femenina de la falange y sus relaciones con los países amigos. Música, danza y política exterior durante la Guerra y el primer franquismo (1937-1943). En: PÉREZ ZALDUONDO, Gemma y CABRERA GARCÍA, Ma Isabel (Coords.). Cruces de caminos. Intercambios musicales y artísticos en la Europa de la primera mitad del siglo XX. Granada: Universidad de Granada, 2010. p. 357-406.

MINISTERIO DE EDUCACIÓN NACIONAL. Circular a la Inspección de Primera Enseñanza y Maestros Nacionales, Municipales y privados de la España Nacional. Boletín Oficial del Estado, Burgos, n. 503, p. 6154-6156, 8 de marzo de 1938a.

MINISTERIO DE EDUCACIÓN NACIONAL. Orden aprobando, con carácter obligatorio, los Programas Escolares para las Escuelas Primarias Nacionales. Boletín Oficial del Estado, n. 172, Burgos, p. 3028, 19 de diciembre de 1938b.

MINISTERIO DE EDUCACIÓN NACIONAL. Orden convocando el primer curso para Maestros de "Orientaciones nacionales para la educación primaria". Boletín Oficial del Estado, n. 574, Burgos, p. 7386-7387, 18 de mayo de 1938c.

MINISTERIO DE EDUCACIÓN NACIONAL. Orden designando una Comisión para formar los programas que han de regir en las Escuelas Nacionales de Primera Enseñanza. Boletín Oficial del Estado, n. 539, Burgos, p. 6763-6764, 13 de abril de 1938d.

MINISTERIO DE EDUCACIÓN NACIONAL. Preámbulo de los Programas Escolares aprobados por Orden Ministerial conteniendo normas y métodos para la educación primaria. Boletín Oficial del Estado, Burgos, n. 172, p. 3035-3036, 19 de diciembre de 1938e.

MINISTERIO DE EDUCACIÓN NACIONAL. Orden autorizando la publicación, venta y uso en las escuelas de Primera Enseñanza de los libros escolares aprobados por la Comisión dictaminadora. Boletín Oficial del Estado, Burgos, n. 81, p. 1647-1649, 22 de marzo de 1939a.

MINISTERIO DE EDUCACIÓN NACIONAL. Orden autorizando un concurso de material pedagógico con destino a las Escuelas Nacionales de Primera Enseñanza. Boletín Oficial del Estado, Burgos, n. 192, p. 3784, 11 de julio de 1939b.

MINISTERIO DE EDUCACIÓN NACIONAL. Orden reorganizando el Patronato de Misiones Pedagógicas. Boletín Oficial del Estado, Burgos, n. 184, p. 3641, 3 de julio de 1939c.

MINISTERIO DE EDUCACIÓN NACIONAL. Orden por la que se establecen en todos los Centros de Primera y Segunda Enseñanza las disciplinas de Educación Política, Física y Deportiva y las de Iniciación en las Enseñanzas del Hogar, bajo la inspección y vigilancia del Frente de Juventudes. Boletín Oficial del Estado, Madrid, n. 291, p. 8090, 18 de octubre de 1941a.

MINISTERIO DE EDUCACIÓN NACIONAL. Orden por la que se reglamenta la creación oficial de Escuelas del Hogar. Boletín Oficial del Estado, Madrid, n. 227, p. 6277, 15 de agosto de 1941b.

MINISTERIO DE EDUCACIÓN NACIONAL. Orden sobre obligatoriedad de las disciplinas de Escuelas del Hogar en la Enseñanza Media. Boletín Oficial del Estado, Madrid, n. 239, p. 6451, 26 de agosto de 1944.

MINISTERIO DE EDUCACIÓN Y CIENCIA. Orden de 17 de enero de 1981 por la que se regulan las enseñanzas de Educación Preescolar y del Ciclo Inicial de la Educación General Básica. Boletín Oficial del Estado, Madrid, n. 18, p. 1384-1389, 21 de enero de 1981a. 
MINISTERIO DE EDUCACIÓN Y CIENCIA. Real Decreto 69/1981, de 9 de enero, de ordenación de la Educación General Básica y fijación de las enseñanzas mínimas para el Ciclo Inicial. Boletín Oficial del Estado, Madrid, n. 15, p. 1096-1098, 17 de enero de 1981b.

MINISTERIO DE EDUCACIÓN Y CIENCIA. Orden de 6 de mayo de 1982 por la que se regulan las enseñanzas del Ciclo Medio de la Educación General Básica. Boletín Oficial del Estado, Madrid, n. 115, p. 12574-12586, 14 de mayo de 1982a.

MINISTERIO DE EDUCACIÓN Y CIENCIA. Orden de 25 de noviembre de 1982 por la que se regulan las enseñanzas del Ciclo Superior de la Educación General Básica. Boletín Oficial del Estado, Madrid, n. 291, p. 33446-33466, 4 de diciembre de 1982b.

MINISTERIO DE EDUCACIÓN Y CIENCIA. Real Decreto 3087/1982, de 12 de noviembre, por el que se fijan las enseñanzas mínimas para el ciclo superior de Educación General Básica. Boletín Oficial del Estado, Madrid, n. 280, p. 32011-32017, 22 de noviembre de 1982c.

MINISTERIO DE FOMENTO. Ley de Instrucción pública autorizada por el Gobierno para que rija desde su publicación en la Península é Islas adyacentes, lo que se cita. Gaceta de Madrid, Madrid, n. 1710, p. 1-3, 10 de septiembre de 1857.

MINISTERIO DE INSTRUCCIÓN PÚBLICA Y BELLAS ARTES. Real orden aprobando las reglas que se insertan, propuestas por la Junta para ampliación de estudios e investigaciones científicas, en cumplimiento de lo prevenido en el Real decreto de 10 de Mayo último, y a las cuales habrá de atenerse el funcionamiento del Instituto Escuela de segunda enseñanza, creado por dicho Real decreto. Gaceta de Madrid, Madrid, n. 199, p. 163-167, 18 de julio de 1918.

MOLERO PINTADO, Antonio. La educación durante la Segunda República y la Guerra Civil (1931-1939). Madrid: Centro de Publicaciones, Ministerio de Educación y Ciencia, 1991. 514p.

MUÑOZ-REPISO, Mercedes et al. El sistema educativo español. Madrid: Centro de Investigación y Documentación Educativa, 2000. 426p.

ORIOL DE ALARCÓN, Nicolás. La música en las enseñanzas de régimen general en España y su evolución en el s. XX y comienzos del XXI. Revista electrónica de LEEME, Valencia, n. 16, nov. 2005. Disponible en: <http://musica.rediris.es/leeme/revista/oriol05.pdf >. Acceso el: 1 de octubre de 2015.

ORTIZ MUÑOZ, Antonio. Reportaje. Más de mil quinientas bibliotecas populares han sido repartidas por el Ministerio de Educación. Revista nacional de educación, Madrid, n. 25, p. 75-78, 1943.

PERALTA ORTIZ, Ma Dolores. La escuela primaria y el magisterio a comienzos del franquismo. Madrid: Universidad Pontificia de Comillas, 2012. 288p.

PÉREZ COLODRERO, Consuelo y GARCÍA GIL, Desirée. Despertando en almas jóvenes un amor profundo por España': estudio del Cancionero de la Sección Femenina del Frente de Juventudes de F.E.T y de las J.O.N.S. (1943). En: COLLADO SEIDEL, Carlos (Ed.). Himnos y canciones: símbolos de identidad colectiva. Granada: Comares, 2016a [en prensa].

PÉREZ COLODRERO, Consuelo y GARCÍA GIL, Desirée. 'España entera al servicio de todo el mundo': música, mujer y educación en el archivo histórico del NODO de RTVE. En: ARCE, Julio (Ed.). La creación musical en la Banda Sonora. Madrid: SEdeM, 2016b [en prensa].

PÉREZ PRIETO, Mariano. La organización de la educación musical en España desde 1970: estudio a partir de los textos legales de ámbito estatal. Aula: Revista de Pedagogía de la Universidad de Salamanca, Salamanca, n. 13, p. 191-214, 2001. 
PÉREZ PRIETO, Mariano. La enseñanza de la Música en la Educación Secundaria en España desde 1970 según los documentos oficiales de ámbito estatal. Revista interuniversitaria de formación del profesorado, Zaragoza, n. 52, p. 77-94, 2005.

PÉREZ ZALDUONDO, Gemma. La música en España durante el franquismo a través de la legislación (1936-1951). Granada: Universidad de Granada, 2002. Recuperado el 14 de septiembre de 2015 de: <http://hdl.handle.net/10481/4427>.

PRESIDENCIA DE LA JUNTA DE DEFENSA NACIONAL. Orden de 19 de agosto de 1936. Acordando que las escuelas nacionales de instrucción primaria reanuden las enseñanzas el día primero del próximo septiembre. Boletín Oficial de la Junta de Defensa Nacional de España, Madrid, n. 9, p. 35-36, 21 de agosto de 1936a.

PRESIDENCIA DE LA JUNTA DE DEFENSA NACIONAL. Orden de 4 de septiembre de 1936. Dictando reglas a las que habrá de sujetarse la enseñanza en los Institutos nacionales durante el próximo curso escolar. Boletín Oficial de la Junta de Defensa Nacional de España, Madrid, n. 18, p. 70-71, 8 de septiembre de 1936b.

PRESIDENCIA DE LA JUNTA DE DEFENSA NACIONAL. Orden de 4 de septiembre de 1936. Ampliando a todos los Centros docentes las reglas contenidas en la Orden de esta fecha relativa a los Institutos nacionales. Boletín Oficial de la Junta de Defensa Nacional de España, Madrid, n. 18, p. 72, 8 de septiembre de 1936c.

PUELLES BENÍTEZ, Manuel. Educación e ideología en la España contemporánea. Madrid: Tecnos, 2010.

RICHMOND, Kathleen. Las mujeres en el fascismo español. La sección femenina de la falange 1934-1959. Madrid: Alianza, 2004.

ROCHE MÁRQUEZ, Elisa. La enseñanza de la música en el marco de la LOGSE. Aula de innovación educativa, Barcelona, n. 24, p. 5-8, 1994.

RUSINEK, Gabriel y SARFSON, Susana. «Spain: a journey from a nominal towards a universally implemented curriculum». En: COX, Gordon y STEVENS, Robin S. (Eds.). The origins and foundations of music education: cross-cultural historical studies of music in compulsory schooling. London, UK: Continuum, 2010. p. 91-108.

SABÍN RODRÍGUEZ, José Manuel. La Dictadura franquista (1939-1975). Textos y documentos. Madrid: Akal, 1997. 447 p.

Consuelo Pérez-Colodrero - Dra. Eu. en Historia y Ciencias de la Música. Grupo de Investigación HUM-263 'Patrimonio Musical de Andalucía'. Licenciada en Historia de la Música, Profesora Superior de Piano, Profesora Superior de Solfeo, Teoría de la Música, Repentización y Transporte. Sus líneas de investigación abarcan el estudio el patrimonio musical andaluz, prestando especial atención a sus aspectos historiográficos e identitarios, y el estudio del curriculum histórico, con especial énfasis en cuestiones sobre música y género. ORCID record: https://www.orcid. org/0000-0001-9995-0417.

Desirée García-Gil - Doctora por la Universidad de Granada con mención de «Doctor Europeus», Licenciada en Teoría de la Literatura, Diplomada en Educación Musical y Profesora Superior de Piano. Sus líneas de investigación abarcan el análisis del curriculum y las relaciones entre música, género y educación. Ha publicado en revistas como Música y Educación, Musiker, Eufonía o Egitania Sciencia. Ha participado en proyectos de investigación competitivos financiados por la Agence National Recherche (Universidad Sorbonne), el MECD (I+D+i) y la Junta de Andalucía. Ha realizado estancias de investigación subvencionadas en Francia e Italia, formando parte de Comités Científicos en Revista de Educación de UCM y Journal for Educators, Teachers and Trainers. Actualmente es profesora en la UCM. 